\title{
Managed probability of Penny series against classical probability series of equal length. Not a typical conversion Mises Filatov $\mathbf{O}$. \\ Управляемая вероятность выпадения серий Пенни против классической вероятности выпадения серий равной длины. Не типичное преобразование Мизеса Филатов О. В.
}

\author{
Филатов Олег Владимирович / Filatov Oleg Vladimirovich - инженер-программист,
} НТЦ Модуль, г. Москва

\begin{abstract}
Аннотация: раскрывается причина образования парадокса вероятностей серий в игре Пенни как следствие правил самой игры и способ управления вероятностью их выпадения. Показана зависимость (относительность) вероятностей выпадения серий любых длин случайных событий, от логических правил их образования из «сырого материала» случайной бинарной последовательности. Даньл алгоритмы, приводящие к нарушению «механизма наследования» между материнской и дочерней последовательностью в случайном преобразовании Мизеса.

Abstract: reveals the reason for the formation of the paradox series of probabilities in the game Penny as a consequence of the rules of the game and the way to control the probability of precipitation. The dependence of (relative) probability of a series of random events of any length, from the logical rules of formation of the "raw material" of a random binary sequence. Are given algorithms, resulting in the disruption of "inheritance" between mother and daughter in a random sequence of transformation Mises.
\end{abstract}

Ключевые слова: эл, элементарное событие, иуг, инверсия, Мизес, Пенни, игра Пенни, нетранзитивность, управление вероятностью, преобразование Мизеса, материнская последовательность, производная последовательность, вторичные последовательности, ошибка лектора, скользящее окно, поисковое окно, поисковый алгоритм, поисковый шаблон, эффект экранирования, НТЦ Модуль.

Keywords: el, elementary event, train, inversion, Mises, Penny, Game Penny, not transitive, probability management, conversion of Mises, the parent sequence, a daughter of the sequence, secondary sequence, lecturer error, sliding window, search window, search algorithm, search pattern, shielding effect, STC Module.

Сокращения: эл - элементарное событие. В статье, элементарное событие имеет значения «0» или «1» и их вероятности выпадения равны 0,5.

Введение

На лекциях по теории вероятности уважаемые лекторы обычно устанавливают контакт с аудиторией через вопрос о том, что чаще встречается в случайной бинарной последовательности: серии из $\mathrm{n}$ нулей подряд «000..00» или серии -«гребёнки» «0101..» такой же длины. При этом демонстрируется и пример случайной пос-ти (длина которой пропорциональна терпению рисующего её лектора): «1001110101000011...».

Контакт с аудиторий считается установленным, когда слушатели либо дают ответ, либо соглашаются с тем, что количество встреч таких серий должно быть примерно поровну, так как выпадение серий равновероятно.

Этот ответ был правильным для уровня знаний о случайных бинарных событиях, до тех пор, пока Уолтер Пенни не открыл нетранзитивность серий из элементарных событий в 1969 году. Эта нетранзитивность получила название «Игра Пенни».

\section{Основная часть}

Отметим, что классические серии игры Пенни имеют длину в три события. В этой статье, из-за ограничений её объёма, рассматриваются именно они. Хотя серии могут быть любой длинны, начиная с длины в два события. Для проведения компьютерных экспериментов удобнее применять термин «шаблон», вместо термина «серия», серии и шаблоны в этой статье обозначают одно и то же.

Под обнаружением выпадения шаблона (серии) в последовательности выпадений монеты, со сторонами «0» и «1», по Пенни подобным правилам игры, понимается система последовательных поисковых переборов, с учётом найденных шаблонов. Поиск по правилам игры Пенни производится скользящим окном, в котором точно помещается поисковый шаблон, например «111». Окно движется вдоль случайной бинарной пос-ти. Содержимое окна на каждом шаге сравнивается с поисковым шаблоном. Когда содержимое окна поразрядно совпадёт с шаблоном, тогда увеличивается на один счётчик найденных шаблонов [2, 3]. 
Пример, «Поисковое окно» («Скользящие окно»). Поисковое окно пошагово движется по бинарной последовательности:

Ша $1 0 1 0 \longdiv { 1 1 0 } 1 1 0 1 1 0 0 0$

На k-ом шаге в поисковом окне находится комбинация «001», которая не совпадает поразрядно с поисковым шаблоном «111».

1, Шаг $1 0 1 0 1 \longdiv { 1 1 } 1 0 1 1 0 0 0$

На k+1 шаге скользящего окна в нём находится комбинация «011», не равная поисковому шаблону «111».

1., Шаг $1 0 1 0 1 1 \longdiv { 1 1 } 0 1 1 0 0 0$ - Найдена искомая

Поисковый шаблон «111» поразрядно совпал с комбинацией в скользящем окне. Увеличивается на один значение счётчика найденных шаблонов. Скользящие окно в шаге $\mathrm{k}+3$ переходит в новую позицию для поиска следующей комбинации «111». В поисковое окно загружаются три следующих элементарных события (эл):

$\underset { 1 , \text { Шаг } } { 1 1 1 } 1 0 1 0 1 1 0 0 0 \longdiv { 1 1 0 } 0 0 0$

Объяснение сути эффекта экранирования одним шаблоном другого шаблона. Обратите особое внимание на шаги: $\mathrm{k}+1$ и k+2. При поиске скользящим окном, серия «011» всегда предшествует серии «111». Более того, правила игры приводят к тому, что серия «011» разрушает серию «111», буквально выдирая из серии «111» две единицы «11», что приводит к сокращению численности логически позиционных серий «111» в пос-ти. Именно правила поиска шаблонов в игре Пенни являются организаторами численного неравенства в большинстве пар конкурирующих друг с другом шаблонов. Для других пар конкурирующих шаблонов так же работает только что описанный эффект экранирования.

В случае поиска не одной, а нескольких комбинаций сразу, содержимое скользящего окна поразрядно и поочерёдно сравнивается с каждым из поисковых шаблонов. И, в случае совпадения, производится увеличение значения счётчика обнаружения конкретного поискового шаблона, а поисковое окно перемещается вправо на три элементарных события (эла), сразу за найденной серией.

Заметим, что любую случайную пос-ть можно образовать из восьми трёхразрядных шаблонов: 000; 001; 010; 011; 101; 110; 111, с точностью до одного, двух разрядов в её конце (при не кратности длины пос-ти трём).

Игра Пенни построена на неявном управлении численностью шаблонов путём их парных комбинаций (двух конкурирующих шаблонов). На длине в три бита, возможны восемь различных комбинаций шаблонов Пенни. Зарезервируем одну комбинацию «000». В таблице 1 показаны результаты компьютерного эксперимента по парной конкуренции шаблона ${ }^{000} \mathrm{Sh}(\mathrm{N})=« 000 »$, по отдельности с каждым из семи возможных шаблонов ${ }^{\mathrm{Xn}} \mathrm{Sh}(\mathrm{N})$. Численности найденных шаблонов ${ }^{\mathrm{Xn}} \mathrm{Sh}(\mathrm{N})$, при конкуренции с «000», преимущественно разные, что невозможно с позиций равной вероятности выпадений комбинаций одинаковой длины.

Например, при $N=2 \cdot 10^{7}$ подбрасываний монеты в компьютерном эксперименте, результатом конкуренции шаблона «000» с «010» явилось обнаружение 1765145 шаблонов «010» (таблица 1). Результатом конкуренции шаблона «000» с шаблоном: ${ }^{\mathrm{Xn}} \mathrm{Sh}(\mathrm{N})=« 100 »$ явилось обнаружение 2498933 шаблонов «100» (таблица 1). При поиске только одного шаблона «000» (без любого другого конкурирующего шаблона), в той же самой в пос-ти, обнаружено 1428478 шаблонов «000». Следовательно, если нужно найти в пос-ти, при её просмотре, разные количества шаблонов, то искомый шаблон (например, шаблон «000») комбинируется с одним из семи других возможных шаблонов. Найденные количества шаблонов ${ }^{\mathrm{Xn}} \mathrm{Sh}(\mathrm{N})$ при их конкуренции с шаблоном ${ }^{000} \mathrm{Sh}(\mathrm{N})=\ll 000 »$, представлены в таблице 1.

Так как целью компьютерного эксперимента было найти по правилам игры Пенни разное количество шаблонов ${ }^{\mathrm{Xn}} \mathrm{Sh}(\mathrm{N})$, то найденные вмести с ними численности конкурирующего им шаблона «000» не показаны в таблице 1. Если пары найденных шаблонов: ${ }^{000} \mathrm{Sh}(\mathrm{N})$ и конкурирующий ему шаблон ${ }^{\mathrm{Xn}} \mathrm{Sh}(\mathrm{N})$ (семь пар) делить на общее число событий каждой пары: ${ }^{000} \mathrm{Sh}(\mathrm{N}) /\left({ }^{000} \mathrm{Sh}(\mathrm{N})+{ }^{\mathrm{Xn}} \mathrm{Sh}(\mathrm{N})\right) ;{ }^{\mathrm{Xn}} \mathrm{Sh}(\mathrm{N}) /\left({ }^{000} \mathrm{Sh}(\mathrm{N})+\right.$ $\left.{ }^{\mathrm{Xn}} \mathrm{Sh}(\mathrm{N})\right)$, то получим известную таблицу выигрышных пропорций Пенни. Но, статья обращает внимание на то, что следует из этой таблицы. А из таблицы следует, что побеждают те комбинации, которых больше (иначе бы они не побеждали с постоянным процентным соотношением). А сочетание разных шаблонов между собой, является способом управления вероятностью обнаружения шаблонов.

Этот способ управления вероятностью обнаружения (выпадения) шаблонов имеет такое же право на существование, какое имеет право на существование нетранзитивный парадокс Пенни (игра Пенни).

Ещё раз опишем, как работает управление вероятностью выпадением шаблонов $\mathrm{ShA}=$ «000» для таблицы 1. Окно пошагово перемещается вдоль случайной бинарной пос-ти. Как только в окне 
появляется комбинация «000», то окно устанавливается «рывком», сразу за ней. По окончании просмотра бинарной пос-ти обнаруженная окном численность шаблона ShA заносится в таблицу 1. Таким же образом регулируется численность обнаружения, и: $\mathrm{ShB}, \mathrm{ShC}, \ldots \mathrm{ShX}$.

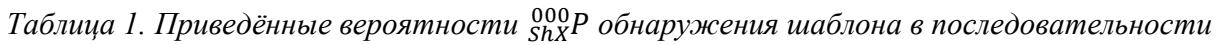

\begin{tabular}{|c|c|c|c|c|c|c|c|c|}
\hline$N=2 \cdot 10^{7}$ & \multicolumn{7}{|c|}{000 - конкурент для шаблонов ShX } & \multirow{2}{*}{000} \\
\hline ShX & 110 & 011 & 101 & 010 & 001 & 111 & 100 & \\
\hline $\operatorname{Sh} X(N)$ & $\begin{array}{r}250 \\
0389\end{array}$ & $11{ }^{21437}$ & $98 \quad 20010$ & $\begin{array}{l}176 \\
5145\end{array}$ & $\begin{array}{l}142 \\
8269\end{array}$ & $\begin{array}{l}142 \\
9154\end{array}$ & $\begin{array}{l}249 \\
8933\end{array}$ & $\begin{array}{r}142 \\
8478\end{array}$ \\
\hline$\frac{N}{\operatorname{Sh} X(N)}$ & 8,00 & 9,33 & 9,99 & 11,3 & 14,0 & 13,9 & 8,00 & 14,0 \\
\hline${ }^{000} f_{\text {ShX }}=\frac{n \cdot \operatorname{ShX}}{N}$ & 0,37 & 0,322 & 0,300 & 0,26 & 0,21 & 0,21 & 0,37 & 0,21 \\
\hline${ }_{\operatorname{ShX}}^{000} P=\frac{{ }^{000} f_{\text {ShX }}}{\sum\left({ }^{000} f_{\text {ShX }}\right)}$ & $5^{0,16}$ & 0,141 & 0,132 & 0,11 & 0,09 & 0,09 & 0,16 & 0,09 \\
\hline
\end{tabular}

В строке: $\frac{N}{\operatorname{ShX}(N)}$, таблицы 1 показано среднее количество элементарных событий (эл), приходящихся на один искомый шаблон.

В строке: ${ }^{000} f_{S h X}=\frac{n \cdot S}{N}$, показана фракционность (f - fraction, часть), какую часть занимает шаблон $\operatorname{Sh} X(N)$ своими элементарными событиями от полной длины случайной пос-ти $N$, при конкуренции с шаблоном ${ }^{000} \mathrm{Sh}(\mathrm{N})$.

В строке: ${ }_{S h X}^{000} P=\frac{{ }^{000} f_{S h}}{\sum\left({ }^{000} f_{S h X}\right)}$ - приведённые вероятности каждой фракции.

Тривиальным шагом является сделать аналогичные таблицы для других комбинаций шаблонов: ${ }_{\operatorname{ShX}}^{001} P ; \underset{\operatorname{ShX}}{010} ;{ }_{\operatorname{ShX}}^{011} P . .$. Ради экономии места опустим их, и перейдем к дальнейшему развитию темы управления вероятностью выпадения шаблонов (в смысле поисковых правил игры Пенни).

В основе более тонкого управления выпадением шаблонов, по правилам игры Пенни, лежит идея дополнительной конкуренция, за счёт ввода к уже имеющемуся шаблону ещё одного или нескольких конкурирующих шаблонов. Так, шаблон «100» сильно экранирует шаблон «000», но если к этой паре добавить любой из шаблонов: «001» и/или «011» (которые совсем ни оказывают никакого экранирующего воздействия на шаблон «000»), то часть шаблонов «100» будет экранировано добавленным шаблоном: «001» и/или «011». Результатом этого явится увеличение количества выпадений шаблона «000». Более тонкое управление количеством (частотой, вероятностью) выпадения шаблона «000», в таблице 2 .

Таблица 2. Регулировка обнаружения «000» экранирующими шаблонами

\begin{tabular}{|c|c|c|}
\hline Искомые шаблоны & $\begin{array}{c}\text { «000» } \\
\text { обнаружений }\end{array}$ & Пояснения \\
\hline 000 & 1428478 & Нет экранирующих шаблонов (э.ш.) \\
\hline $000 ; 110$ & 1072514 & 110 - э.ш. для 000 \\
\hline $000 ; 110 ; 111$ & 1225339 & 110 - э.ш. для: 000; 111 - э.ш. для: 110 \\
\hline $000 ; 110 ; 111 ; 100$ & 613148 & $\begin{array}{l}\text { 110, } 100 \text { - э.ш. для: 000; 111- э.ш. для: 110,100; } \\
\text { 110- э.ш. для: } 100\end{array}$ \\
\hline $\begin{array}{l}000 ; 110 ; 111 ; 100 ; \\
001\end{array}$ & 667348 & --//--; 001 - э.ш. для 100 \\
\hline $000 ; 110 ; 100$ & 536328 & 110, 100 - э.ш. для: 000; 110 - э.ш. для 100 \\
\hline $000 ; 100$ & 357814 & 100 - э.ш. для: 000 \\
\hline
\end{tabular}

В строке 1 таблицы 2 численность обнаруженных в эксперименте Пенни - способом, шаблонов «000», равно 1428478 . 
При добавлении в список поиска программы экранирующего шаблона «110» («000»+ «110») и одновременном поиске двух шаблонов, число найденных шаблонов «000» уменьшилось до 1072514 (строка 2).

Строка 3 соответствует эксперименту, в список поиска которого добавлен третий шаблон: «111». При одновременном поиске в пос-ти трёх шаблонов, шаблон «111» является экранирующим (киллером) для шаблона «110», и экранирует (убивает) их некоторое количество. За счёт этого снимается экранирование с шаблона «000» и его численность возрастает до 1225339.

В строке 4, к группе конкурирующих шаблонов добавлен новый экран «100» (самый сильный) для шаблона «000». В результате находимая численность «000» уменьшилась до 613148.

В строке 5, для ещё более тонкой регулировки, добавлен шаблон «001», который является экранирующим шаблоном для «100». В результате одновременного поиска по всем шаблонам, численность шаблона «000» увеличилась, было найдено 667348 шаблонов «000».

В строке 6 уменьшение численности находимых «000» шаблонов достигнуто за счёт присутствия в поисковом списке двух экранирующих его шаблонов: «110», «100». Так как шаблон «110» является экранирующим для шаблона «100», то численность «000» была уменьшена не до минимально возможной.

В строке 7 достигнута минимально возможная численность шаблона «000» для этого способа поиска, за счёт применение одного экранирующего шаблона «100» (самого сильного экрана для шаблона «000»).

Очевидно, что идея тонкой регулировки численности за счёт комбинации разных экранирующих шаблонов применима не только к рассмотренным в таблице 2 шаблонам, но к любым шаблонам, любой длины.

Теперь рассмотрим утверждение, приведённое в разделе «введение» этой статьи, что уважаемые лекторы ошибаются, утверждая равную вероятность выпадения «гребёнок» («0101», «1010») и монотонных событий («0000», «1111»). Для конкретности рассмотрим шаблоны из четырёх эл, таблица 3.

Таблица 3. Ошибка уважаемых лекторов, после 1969 года

\begin{tabular}{|c|c|c|c|}
\hline & Шаблоны & Найдено шаблонов & Примечание \\
\hline 1 & $\begin{array}{l}\ll 0000 » \\
\ll 0101 »\end{array}$ & $\begin{array}{l}667039 \\
934404\end{array}$ & \multirow{2}{*}{$\begin{array}{l}\text { Шаблон «1010» является слабо экранирующим } \\
\text { для шаблона «0000», поэтому в строке } 2 \text { шаблонов } \\
\text { «0000» меньше, чем в строке } 1 \text {. }\end{array}$} \\
\hline 2 & $\begin{array}{l}\ll 0000 » \\
\ll 1010 »\end{array}$ & $\begin{array}{l}600563 \\
1000221\end{array}$ & \\
\hline 3 & $\begin{array}{l}\text { «1111» } \\
\ll 1010 » \\
\end{array}$ & $\begin{array}{l}666146 \\
932755 \\
\end{array}$ & \multirow{2}{*}{$\begin{array}{l}\text { Шаблон «0101» является слабо экранирующим } \\
\text { для шаблона «1111», поэтому в строке } 4 \text { шаблонов } \\
\text { «1111» меньше, чем в строке } 3 \text {. }\end{array}$} \\
\hline 4 & $\begin{array}{l}\ll 1111 » \\
\text { «0101» }\end{array}$ & $\begin{array}{l}599644 \\
1000214\end{array}$ & \\
\hline & пар шабло & зведён по правилам I & 3 пос-ти из $N=2 \cdot 10^{7}$ эл \\
\hline
\end{tabular}

Уважаемые лекторы рассматривают именно пары шаблонов одинаковой длины, но именно рассматриваемые пары шаблонов встречаются с разной частотой (вероятностью) при поиске по правилам игры Пенни. Можно возразить, что лекторы имели в виду другие способы поиска, но тогда, лекторы должны были бы рассказать об этих способах поиска. Указать, по какому из них указанные события находятся с равной вероятностью, а при каких способах нет.

В качестве справки укажем, что в потоковом способе поиска $[1,2,3]$ численность последовательных шаблонов длины $\mathrm{n}$ («111..») рассчитывается по формуле: ${ }^{n} S X=\frac{N}{2^{n+2}}$, а численность инверсных шаблонов длины $\mathrm{n}(« 1010 \ldots »)$ рассчитывается по ф-ле: ${ }^{1} C_{n}=\frac{N}{2^{n+4}}$, т.е. численности различаются в четыре раза.

Парадокс нетранзитивности в игре Пенни не укладывается в привычное мировоззрение, сформированное доминантной версией теории вероятности, которая базируется на ф.1, расчёта числа выпадений комбинаций из n бинарных событий (серий длины n) при N бросках монеты:

$$
{ }^{n} S_{N}=\frac{N}{n \cdot 2^{n}}
$$

Деформации составных событий при фрагментации бинарной пос-ти на участки равной длины рассмотрены в работе [4]. Для примера рассчитаем ожидаемое число выпадений любой комбинации из трёх элементарных событий («100»;«011»;«001»; «110»; «111»; «000»; «101»; «010») при $N=2 \cdot 10^{7}$ бросков монеты. По ф.1: ${ }^{n=3} S_{N}=\frac{2 \cdot 10^{7}}{3 \cdot 2^{3}}=833333$. В таблице 4, в подразделе «Раздельный поиск комбинаций в фрагментах длиной $\mathrm{n}=3$ (R2)» приведены результаты поиска этих комбинаций в $N=2 \cdot 10^{7}$ бросках монеты. 
Для объяснения парадокса Пенни, ф.1 не подходит. Парадокс Пенни объясняется с помощью экранирования поисковыми шаблонами друг друга. Вывод формул для численного расчёта получаемых результатов в пос-ти из $N$ бросков монеты при поиске шаблонов по правилам Пенни, приведён в [7]. Неравенство вероятностей в выпадениях пар конкурирующих шаблонов Пенни приводит к простой (но совершенно некорректной, с точки зрения господствующей теории вероятности) идеи. А именно, если из двух поисковых шаблонов в игре Пенни, один шаблон побеждает с ощутимым перевесом другой шаблон, то, значит, первый поисковый шаблон чаще встречается в случайной последовательности, чем второй поисковый шаблон.

Разные поисковые шаблоны встречаются, по отдельности, в разных количествах в случайной бинарной последовательности (таблица 4, раздел «Раздельный поиск комбинаций по правилам игры Пенни (R1)»), если их искать по правилам игры Пенни, которые описаны в [7]. В разделе R1, таблицы 4, сгруппированы результаты поиска восьми шаблонов в случайной бинарной пос-ти. Поиск каждого шаблона осуществлялся отдельно от поисков других шаблонов, то есть, каждый поисковый шаблон не имел поисковых конкурентов (конкурирующих шаблонов). Парадоксальным фактом явилось обнаружение разных частот встреч (разные численности) шаблонов имеющих одинаковую длину. Причём, Число встреч шаблона в пос-ти зависит от числа инверсий внутри него (инверсия - это переход от «0» к «1» и, от «1» к «0»). Более подробно о связи числа инверсий внутри группы шаблонов с численностью этих шаблонов в пос-ти описано в [7].

Таблица 4. Результат разных правил $R$ поиска одиночного шаблона

\begin{tabular}{|l|c|c|c|}
\hline Вид шаблонов & «11»; «000» & «100»;«011»;«001»; «110» & «101»; «010» \\
\hline Инверсий в шаблоне & 0 & 1 & 2 \\
\hline Раздельный поиск комбинаций по правилам игры Пенни (R1). & $2000318 ;$ \\
\hline $\begin{array}{c}\text { Найдено шаблонов: } \\
n=3 S_{N}(R 1)\end{array}$ & $1426539 ;$ & $2491065 ; \quad 2499486 ; \quad 2501064 ;$ & 2001218 \\
\hline Эл на шаблон & 1428865 & 8 & 10 \\
\hline Раздельный поиск комбинаций в фрагментах длиной n=3 (R2). & $833712 ; \quad 832724 ; \quad 832299 ;$ & $834538 ;$ \\
\hline $\begin{array}{l}\text { Найдено шаблонов: } \\
n=3\end{array} S_{N}(R 2)$ & $833264 ; 834324$ & 833617 & 832188 \\
\hline
\end{tabular}

Таблица 4 содержит разные численности нахождений одного и того же поискового шаблона (разделы R1 и R2), в одной и той же случайной пос-ти, что немыслимо с позиций равновероятностного выпадения серий (шаблонов). Например, шаблону «111» одновременно принадлежат два разных числа его обнаружений в одной и той же пос-ти: 1426539 (найден по правилам R1), 833262 (найден по правилам R2). Такая двойственная ситуация напоминает ситуацию неопределённости в физике с частицами, которые демонстрируют двойственность своей природы.

Теперь теоретически рассчитаем численность для каждого из поисковых шаблонов, найденных по правилам игры Пенни R1 [7], таблица $4\left(N=2 \cdot 10^{7}\right.$. Обратите внимание, что будут рассчитываться не пары конкурирующих друг с другом шаблонов, а число встреч одного шаблона (без всякой конкуренции со стороны других шаблонов). Для шаблонов «101», «010» учтём, что они являются цугами ${ }^{1} C_{w}$ первой моды [1- 4], применим формулу цуг из [7, ф.8] и таблица 6: ${ }^{1} C\left(R_{1}\right)_{w=3}=\frac{3 \cdot N}{2^{w+2}-3+(-1)^{w+1}}=\frac{3 \cdot 2 \cdot 10^{7}}{2^{3+2}-3+(-1)^{3+1}}=\frac{6 \cdot 10^{7}}{2^{5}-3+1}=2000000$. Теоретически рассчитанная величина мат. ожидания выпадения каждого из шаблонов «101», «010» хорошо совпала с экспериментально обнаруженными количествами этих шаблонов в пос-ти (таблицы 4, 5 - «сумма “Ш”»).

Теоретически рассчитаем численность для каждого из шаблонов, найденных по правилам Пенни R1 [7] в таблице $4\left(N=2 \cdot 10^{7}\right): « 100 », « 011 », « 001 », « 110 »$. Учтём, что эти шаблоны являются краевыми (оконечными) фрагментами последовательных событий [1-3], и применим формулу [7, ф. 10] и таблица 6: $S X_{n=3}=\frac{N}{2^{n}}=\frac{2 \cdot 10^{7}}{2^{3}}=2500000$. Теоретическая величина хорошо совпала с экспериментально обнаруженным количеством таких шаблонов (таблицы 4, 5 - «сумма «Ш»).

Теоретически рассчитаем численность для каждого из шаблонов, найденных по правилам Пенни R1 [7], в таблице $4\left(N=2 \cdot 10^{7)}\right.$ : «111», «000». Эти шаблоны являются фрагментами последовательных событий [1-3]. Применим формулу [7, ф. 13] и таблица 6: $S X_{L=3}=\frac{1}{2} \cdot \frac{N}{2^{L}-1}=\frac{1}{2} \cdot \frac{2 \cdot 10^{7}}{2^{3}-1}=1428571$, что хорошо совпало с обнаруженным количеством поисковых шаблонов в пос-ти (таблицы 4, 5 - «сумма «Ш»). 
Стратегия противодействия слабого шаблона сильному шаблону в игре Пенни. В качестве слабого шаблона возьмём «001» (вероятность победы 1/4), а сильного - «100» (вероятность победы 3/4). Слабый шаблон должен разорвать обманные условия игры, заявив, что он будет получать события для себя от выпадений второй, его собственной, монеты. То есть, одновременно будут подбрасываться две монеты, выпадения одной всегда связаны с поставкой событий для шаблона «001». Выпадения второй монеты всегда связаны с поставкой событий для шаблона «100».

Либо бросается одна монета, но два раза. Один раз для получения события для шаблона «001». Второй раз для получения события для шаблона «100».

В обоих случаях шансы на выигрыш у шаблонов уравниваются -1/2. Пример: число раздельных подбрасываний в эксперименте для каждого из шаблонов $N=2 \cdot 10^{7}$, найдено поисковых шаблонов: «001» - 2501064, «100»- 2499893.

Объяснения получения результата: «001» - 2501064, «100» - 2499893. В таблице 4 дана группировка поисковых шаблонов Пенни в зависимости от числа инверсий в шаблоне. Видно, что шаблоны, принадлежащие одной группе, при раздельном поиске (ищется только этот шаблон) встречаются равновероятно, то есть с вероятностью $1 / 2$. Но, в таблице 4 , есть строка «Эл на шаблон», в которой рассчитано среднее число элементарных событий приходящихся на выпадение одного шаблона. Очевидно, вероятность выпадения шаблонов группы тем выше, чем меньше выпавших эл приходится на любой один шаблон из группы. Формулы расчёта для чисел обнаружения поисковых шаблонов в каждой группе (для раздельного поиска каждого шаблона), даны в таблице 6 (найденные количества поисковых шаблонов, в таблице 4).

Ранее считалось, что для последовательности выпадений монеты допустимы пари на любые комбинации, так как выпадение монеты никак не связаны друг с другом. Из выше сказанного следует, что случайная бинарная пос-ть является более связанным объектом, чем представлялось ранее, и не все возможные условия пари будут честными. Число найденных поисковых шаблонов в бинарной пос-ти зависит от: вида шаблона и от способа его поиска $[5,6]$. Например, есть два игрока. Требуется найти в бинарной пос-ти цепочки состоящие из $n$ единиц или нулей подряд. Победит тот, кто найдёт больше таких цепочек. Первый игрок выбрал стратегию деления последовательности на участки равной длины, число найденных им участков ${ }^{n} S_{N}$ рассчитывается по удвоенной ф.1. Второй игрок выбрал геометрическую стратегию поиска серий $[2,6]$, число найденных им серий ${ }^{n} S_{\mathrm{g}}(N)$ рассчитывается по ф. 2:

$$
{ }^{n} S_{\mathrm{g}}(N)=\frac{N}{k} \cdot \frac{n}{2^{n+1}}
$$

Где $k$ - шаг между погружениями зонда [2, 4, 6].

Сравним численности серий найденных по этим двум стратегиям, разделив ф.2 на ф.1: $\frac{N}{k} \cdot \frac{n}{2^{n+1}}: \frac{2 N}{n \cdot 2^{n}}=$ $\frac{n^{2}}{4 k}$. При: $n^{2}>4 k$ второй игрок всегда выигрывает.

В рамках «не честных пари» кратко упомянем о конкуренции шаблонов разных длин (два шаблона и более). Пусть два игрока играют по правилам игры Пенни, за тем исключением, что выбраны шаблоны «11» и «110». Игрок с шаблоном из трёх событий «110» не одержит ни одной победы над игроком с шаблоном из двух событий «11», если после очередного выигрыша «11» перемещение окна вправо производится на длину минимального шаблона («11»), два эла. Такой экранирующий эффект шаблона «11» для шаблона «110» можно смело назвать «отсекающим» или «запирающим» [1].

Исследование распределений расстояний между выпавиими шаблонами. Были проведены компьютерные эксперименты по выявлению числа элементарных событий выпадающих от окончания шаблона до начала следующего шаблона одного и того же вида. То есть, искались расстояния в элах, разделяющие шаблоны одного и того же вида. Примеры: «11101000111»- между двумя шаблонами вида «111» расстояние пять эл; «111111»- между двумя шаблонами «111» расстояние ноль эл. Распределение расстояний между шаблонами оформлено в виде графиков на рисунках 1,2. Каждая кривая рисунков соответствует нескольким шаблонам, на графиках они совпали в одну линию.

По оси X отложены расстояния между шаблонами (например: от конца шаблона «111» до начала другого шаблона «111»).

По оси Y отложено сколько раз данное расстояние между шаблонами (например «111») было найдено.

На рисунке 1, на графике «Короткие длины», показано распределение коротких промежутков между шаблонами. 


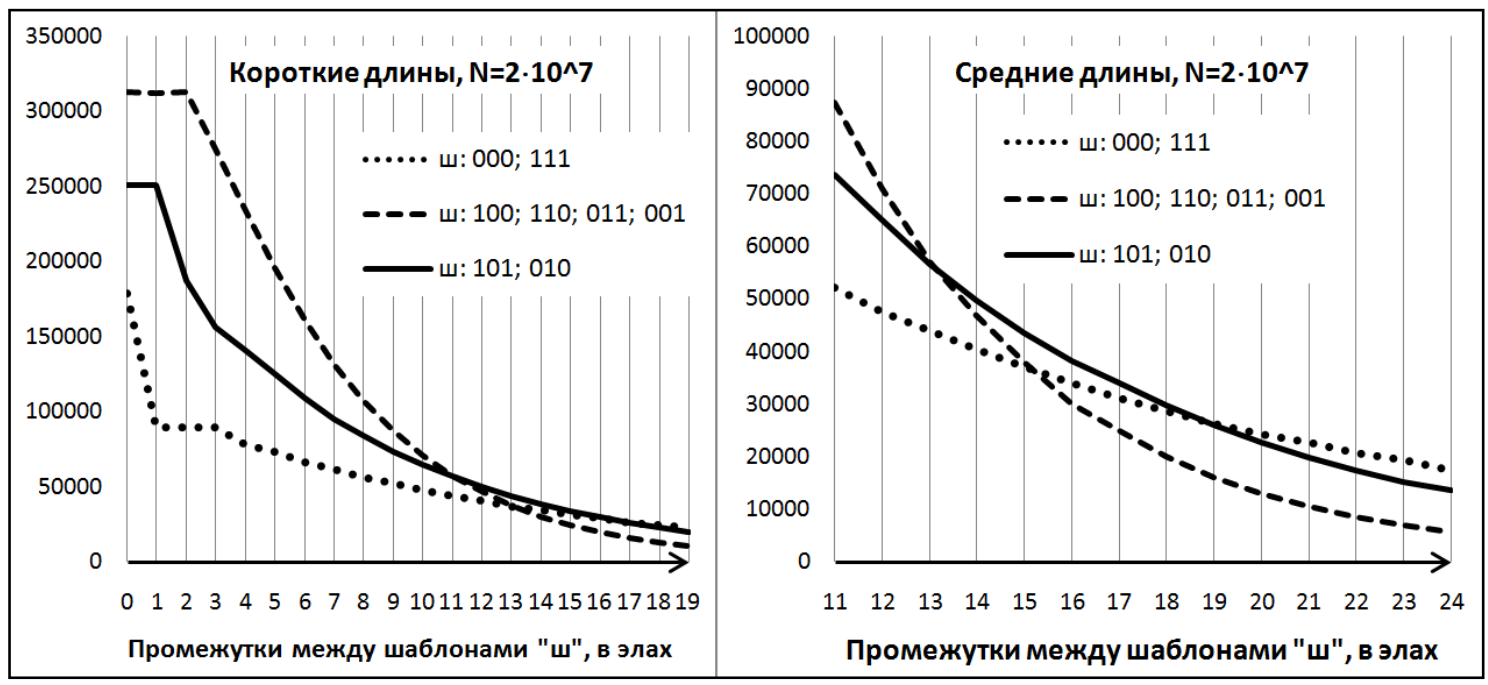

Puc. 1. TabSheetơutton559

Шаблоны: «000» и «111» самые малочисленные (таблица 4, раздел R1), но они способны удаляться друг от друга на большие дистанции, чем шаблоны других двух групп. Начина с удаления в 20 элементарных событий (эл) друг от друга их численность доминирует, рисунок 1 «Средние длины».

На рисунке 1, «Средние длины», выделяется треугольник образованный графиками групп шаблонов. Положение этого треугольника не фиксировано, он перемещается вдоль оси $\mathrm{X}$ в зависимости от числа эл (N) пос-ти. Для приведенных графиков $N=2 \cdot 10^{7}$. Верхняя сторона треугольника образованной сплошной кривой, принадлежащей шаблонам с двумя инверсиями внутри [7], таблица 5. Только на этом участке 14 - 18 эл, они доминируют (затем уходят на промежуточную позицию). Вторая сторона образована графиком шаблонов с одной инверсией внутри. Третья сторона образована графиком шаблонов без внутренних инверсий.

На рисунке 2, «Большие длины», даны продолжения графиков рисунка 1, для длин больше двадцати двух эл.

Таблица 5. Цуги ${ }^{n} C_{w}$ шаблонов “Ш” $\left(N=2 \cdot 10^{7}\right)$

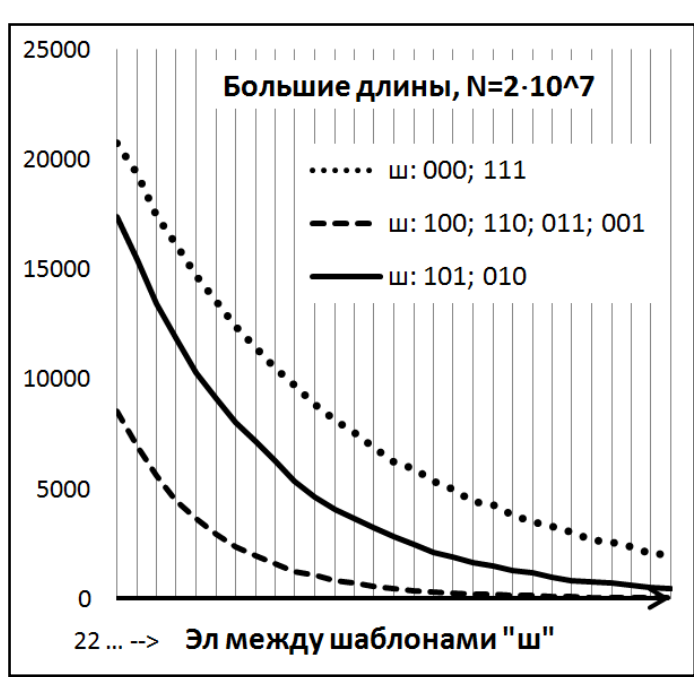

Рис. 2. Большие длинь

\begin{tabular}{|c|l|l|l|}
\hline \multirow{2}{*}{$\begin{array}{c}\text { Число полуволн } \\
\text { в вуге: }\end{array}$} & ш: “000” & ш: “100” & ш: “101” \\
\hline 1 & 0 инверсий & 1 инверсия & 2 инверсии \\
\hline 2 & 1093987 & 1914762 & 1530748 \\
\hline 3 & 136669 & 239384 & 191278 \\
\hline 4 & 17087 & 29919 & 24295 \\
\hline 5 & 2167 & 3746 & 2970 \\
\hline 6 & 276 & 481 & 390 \\
\hline 7 & 26 & 52 & 44 \\
\hline С $0={ }^{n} C_{0 N}$ & 1250222 & 2188355 & 1749730 \\
\hline сумма “Ш”, ${ }^{n} S_{N}$ & 1428865 & 2501065 & 2000318 \\
\hline Эл: $n \cdot{ }^{n} S_{N}$ & 4286595 & 7503195 & 6000954 \\
\hline Эл / Ш: $N /{ }^{n} S_{N}$ & 14 & 8 & 10 \\
\hline
\end{tabular}

Из графика рисунка 2 видно, что шаблоны группы ${ }^{i=0} L(N)(« 000 », \ll 111 »)$, где $(i=0)-$ число инверсий в шаблоне, удаляются друг от друга на расстояния не доступные двум другим группам 
шаблонов, у которых $i>0$. Начиная с некоторого $L$, вероятность того, что расстояние между шаблоном группы $i=0$ становится максимально $(\rightarrow 1)$.

Привести графики для шаблонов иных длин не позволяет размер статьи. Получение этих графиков, аналогично получению уже приведённых графиков.

В работах $[1,2,4]$ вводилось понятие цуги. Существует алгоритм, который с помощью формулы расчёта цуг, для составных событий $[1,2,3]$, позволяет получать псевдослучайную последовательность любой практической длины $[2,8]$. Распределение цуг для шаблонов Пенни представлено в таблице 5. Отличие цуг для шаблонов Пенни от цуг приведённых в [1 -4] в том, что цуги шаблонов Пенни не образованны из составных событий.

Первые полуволны цуг - Пенни, одного вида, имеют между собой коэффициент пропорциональности равный восьми (два в кубе): $8=2^{\mathrm{n}}=2^{3}$. Поскольку между полуволнами (коленами) цуг - Пенни такой же коэффициент, как и между цугами составных событий $[1-4]$, то можно использовать формулы цуг составных для расчёта цуг - Пенни. Для этого используем [4, выкладка 6]: ${ }^{n} C_{0 N}={ }^{n} S_{N}-\frac{{ }^{n} S_{N}}{2^{n}}$, где величина ${ }^{n} S_{N}$ - рассчитывается в зависимости от числа инверсий, для каждой группы шаблонов, таблица 6.

Таблица 6. Формуль расчёта встреч шаблонов (от их вида) и иуг ${ }^{n} C_{0 N}$

\begin{tabular}{|c|c|c|c|}
\hline 1 & 2 & 3 & 4 \\
\hline $\begin{array}{c}\text { Число } \\
\text { инверсий }\end{array}$ & Шаблоны & Формулы для: «сумма “Ш”» из таблицы 5 & $\begin{array}{c}{[4, \text { выкладка 6]: }} \\
n=3\end{array} C_{0 N}={ }^{n} S_{N}-\frac{{ }^{n} S_{N}}{2^{n}}$ \\
\hline 0 & $\begin{array}{c}\ll 111 », \\
\ll 000 »\end{array}$ & $S X_{L=3}=\frac{1}{2} \cdot \frac{N}{2^{L}-1}={ }^{n} S_{N}$ & 1250000 \\
\hline 1 & $\begin{array}{c}\ll 100 », \\
\ll 011 » \\
\ll 110 »\end{array}$ \\
\hline & $\begin{array}{c}\ll 101 », \\
\ll 010 »\end{array}$ & $S X_{n=3}=\frac{N}{2^{n}}={ }^{n} S_{N}$ & 2187500 \\
\hline 2 & ${ }^{1} C\left(R_{1}\right)_{w=3}=\frac{3 \cdot N}{2^{w+2}-3+(-1)^{w+1}}={ }^{n} S_{N}$ & 1750000 \\
\hline
\end{tabular}

В столбце 4, таблицы 6, приведены теоретически рассчитанные мат. ожидания нулевых цуг $\square=3 \square_{0 \square}$, для шаблонов с из трёх эл: $\square=3$. Сравнивая полученные в компьютерном эксперименте значения, строка С0 таблицы 5 , с соответствующими теоретически рассчитанными значениями, столбец 4 таблицы 6 , видим хорошее совпадение экспериментальных и теоретических данных.

Формула, связывающая число нулевых цуг С0 ([4, выкладка 6]) с цугой первой моды [4, ф. 21]: $\square_{\square=1}=\square_{0 \square} \cdot \frac{2^{\square}-1}{2}$. Следовательно, расчёт цуг шаблонов Пенни, с числом полуволн w, рассчитывается по ф. 2.1:

$$
\square \square_{\square}=\frac{\square \square_{1}}{2^{\square-1}}=\square_{0 \square} \cdot \frac{2^{\square}-1}{2^{\square}} \cdot \frac{1}{2^{\square \cdot(\square-l)}}=\square_{0 \square} \cdot \frac{2^{\square}-1}{2^{\square \square}}
$$

Для примера рассчитаем мат. ожидание по ф.2.1 для выпадения цуги $\square=3 \square \square=2$ шаблона Пенни «101» в пос-ти из $\square=2 \cdot 10^{7}$ бросков монеты. Цуга $\square=3 \square_{\square=2}$ этого шаблона выглядит так: «101101». Расчёт проводится по следующим трём пунктам:

1) Рассчитывается число $\square_{\square}$ поисковых шаблонов «101» во всей пос-ти;

2) Рассчитываются нулевые цуги $\square \square_{0 \square}[1-4]$, поискового шаблона «101»;

3) Применяется ф.2.1 с числом полуволн Пенни: $\square=2$. Обратите внимание, что $\square$ - это не число полуволн составных событий, а число шаблонов выпадающих друг за другом.

Выполняя первый пункт, определяем, что в шаблоне две инверсии, поэтому применим формулу из строки «2», таблицы 6: $\square \square \square=\frac{3 \cdot \square}{2^{\square+2}-3+(-1)^{\square+1}}=2000000$.

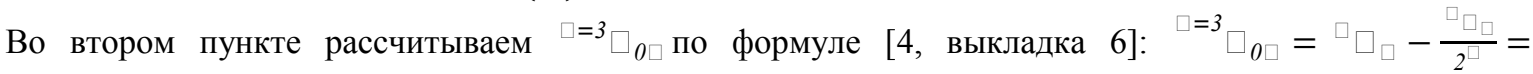
$\square_{\square} \cdot \frac{2^{\square}-1}{2}=1750000$. 
Третьим пунктом применяем $\quad$ ф.2.1: $\quad{ }^{=3} \square_{\square=2}={ }^{\square=3} \square_{0 \square} \cdot \frac{2^{3}-1}{2^{3 \cdot 2}}=191406,25$. Рассчитанное мат. ожидание хорошо совпадает с экспериментально полученным значением (191278) из таблицы 5.

Проведённые выше преобразования и расчёты были произведены в рамках формирующейся «Комбинаторики длинных последовательностей».

Не типичное мизесовское преобразование. Мизес заметил, что из коллектива (так Мизес называл случайную пос-ть) методом «слепого» отбора из неё членов можно образовать только пос-ть, которая сохраняет свойства первичной (материнской) пос-ти. Такое образованием производных (дочерних) постей будем называть преобразованием Мизеса. Не наследование (искажение) свойств материнской последовательности, производной последовательностью, нарушает однозначность мизесовского преобразования. Наследуемыми свойствами являются пропорции составных событий, цуг [1-4] материнской последовательности. В преобразовании Мизеса производная последовательность (дочерняя) всегда наследуют свойства материнской последовательности.

В преобразовании Мизеса применяется «слепой» отбор членов материнской пос-ти. То есть, известны только номера позиций - эл, в которых содержатся случайные значения: «0», «1». Для получения случайного значения из материнской пос-ти, номер его позиции выбирается абсолютно любым образом. Номер может быть рассчитан по любой формуле или получен случайным образом. Номер может быть вычислен по полученным ранее случайным значениям из материнской пос-ти, или без их учёта. Важно только то, что величина («0» или «1») находящаяся в позиции под этим номером, неизвестна. И из материнской пос-ти извлекается эл с неизвестным содержанием («0», «1»). Затем эл, оставаясь неизвестным, может быть вставлен в любую позицию дочерней пос-ти. Если эл становится известным, то он устанавливается в конец производной пос-ти.

Каждое отбираемое в тёмную случайное событие не может быть отбраковано или выбрано по критерию его равенства нулю или единище. Если допустить отбраковку по критерию «0»- «1», то все «0» можно отбросить и создать из «1» не случайную дочернюю пос-ть. Выбирая из «0», «1» можно образовывать серии в дочерней пос-ти по n нулей и по $\mathrm{n}$ единиц, и чередовать их, результатом будет явно не случайная производная пос-ть. Поэтому, каждое отобранное в тёмную случайное событие должно так же «в тёмную» вставляться в любую позицию образуемой производной последовательности, либо в её конец при известном эле.

Производная последовательность (дочерняя) принимает только один экземпляр (эл) каждого случайного события отобранного в тёмную из материнской последовательности, которое обязательно удаляется из материнской пос-ти.

Анализ правил мизисовского преобразования показал, что возможны не противоречащие его правилам построение производной пос-ти, свойства которой не равны свойствам материнской последовательности. То есть, существует не типичное мизесовское преобразование. Нарушение в преобразовании Мизеса достигается применением геометрической вероятности [6] к материнской пос-ти.

Опишем подробнее алгоритм не типичного мизесовского преобразования. Проводилось внедрение зонда, шириной Z, в материнскую последовательность [2, 4]. Все элементарные события, которые «накрыл» собой зонд, записывались в конец создаваемого дочернего файла. Зонд начинал поиск, методом «скользящего вправо (в будущее) окна», описанного в начале статьи, до первой инверсии. Все элы обнаруженные зондом до инверсионного перехода дописывались в порядке их обнаружения в создаваемый дочерний файл.

Поясняющий пример формирования дочерней последовательности. I 72 .

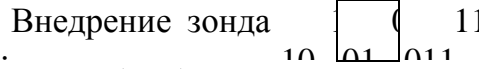

События (элы) из окна записываются в дочернюю пос-ть.

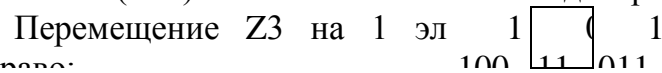

В строке 2 в зондовое окно вошла новая «1», которая была дописана в дочернею пос-ть. Поскольку инверсия в этом шаге не была обнаружена, то осуществляется перемещение зондового окна на один шаг вправо.

$$
\text { Перемещение Z3 на } 1 \text { эл } 1 1 \longdiv { \Omega _ { 1 1 } }
$$

В строке 3 в окно вошла новая «1», которая была дописана в дочернюю пос-ть.

$$
\text { Перемещение Z3 на } 1 \text { эл } 110
$$

В строке 4 в обнаружена инверсия «10». Инверсионное событие («0») не пишется в дочернюю пос-ть. Зонд «извлекается» из материнской пос-ти, перемещается на k позиций вправо (чтобы избежать 
вторичного попадание в одно и то же составное событие), и внедряется в материнскую пос-ть (далее см. строку один).

Полученные выше описанным способом дочерние пос-ти анализировались на долевое содержание в них составных событий и цуг [1-4]. При анализе обнаружено не типичное мизесовское преобразование, заключающееся в том, что производные последовательности не унаследовали распределение составных событий и цуг материнской пос-ти. Графики пропорций составных событий, полученные для ширины зонда в один эл (z1), два эла (z2) и три эла (z3), и график пропорций составных событий в материнской (исходной) пос-ти, приведены на рисунке 3.

Вторичные последовательности образованны из эл, полученных при внедрении зонда Z в материнскую

последовательность, с шагом 50 эл. Число элементарных событий (эл) в каждой из четырёх производных пос-тей 800257 эл. Средняя длина составного события $\mathrm{S}$ в материнской пос-ти: «Исходная пос-ть» - 2,00 эла. Средняя длина составных событий $\mathrm{S}$ в производных пос-тях: «Z3 пость» - 2,67 эла $(\mathrm{S}=300084) ; \ll \mathrm{z} 2$ пос-ть» - 3,00 эла $(\mathrm{S}=266442)$; «z1 пос-ть» - 4,00 эла $(\mathrm{S}=200395)$.

Из рисунка 3 видно, что сильнее всего нарушены пропорции составных событий у дочерней пос-ти, полученной при помощи зонда единичной ширины z1. При дальнейшим росте ширины зонда $(\mathrm{z} 2, \mathrm{z} 3, \ldots)$ пропорции составных событий в дочерних пос-тях приближаются $\quad$ к пропорциям материнской пос-ти.

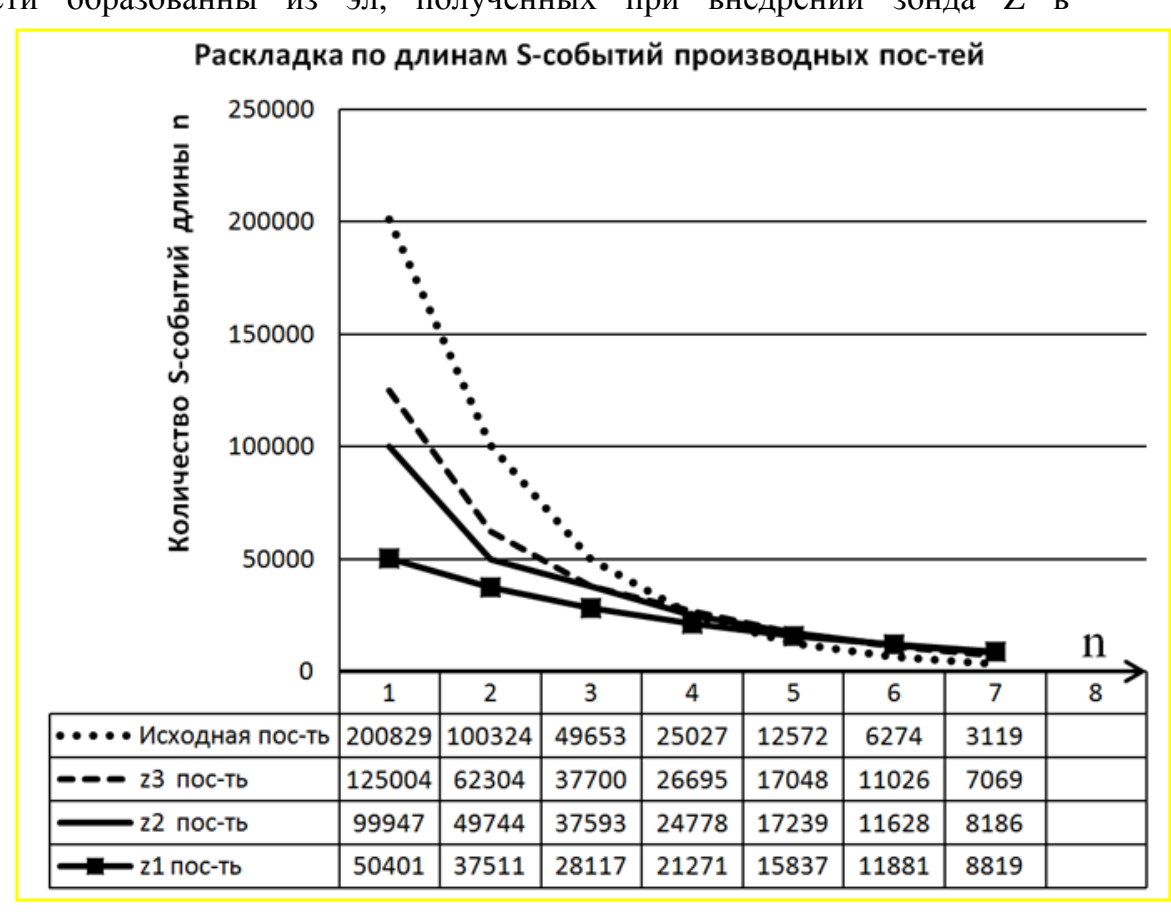

Рисунок 3. «Количество S-событий длины n »

Рис. 3. Количество S-событий длины $n$

\section{Обсуждения}

Не типичное мизесовское преобразование. Правила получения «генетически» иных пос-тей, чем материнская пос-ть, включают фиксацию инверсии меду двух элементарных событий («0»/«1»; «0»/«1»). Описанный выше поисковый алгоритм удовлетворяет требованию мизесовского преобразования, так как оставляет величины инверсных эл неизвестными (неопределёнными). Эл, принёсший инверсию, не заносится в дочернюю пос-ть, он отбрасывается. Значение отбрасываемого эла («0» или «1») остаётся неизвестным. Поэтому эта отбраковка не противоречит отбраковке «вслепую». Так же неизвестно, сколько эл, равных отбракованному элу, находятся в дочерней пос-ти, так как нет учёта и передачи информации о полярности эл от предыдущих зондируемых участков к последующим участкам. Знание о том, что произошёл инверсный переход и применение этого знания в качестве отбраковывающего критерия, является тем фактором, который приводит к «генетическим» нарушениям в «геноме» дочерних пос-тей (по отношению к материнскому «геному»). Автору не удалось найти материалы по применению инверсий для получения нетипичных мизесовских преобразований, и упоминание об инверсиях для этих целей вообще.

Рассмотрим движение поискового окна после его внедрения в качестве зонда в материнскую последовательность (геометрическая вероятность, [6]). Движения окна равноправны в левую и правую стороны пос-ти. Дочерние пос-ти, получаемые описанным в этой статье способом из движений зонда (либо в левую, либо в правую сторону), должны иметь одинаковые процентные распределения своих составных событий. Т.е. не должны зависеть от направления движения зонда. Дочерняя пос-ть не может знать, с какой стороны от зонда берутся элы для её создания, из материнской пос-ти. Но, в зондовой системе координат (в ней есть начало пос-ти и координаты зонда) это очевидно. 
Опишем ещё четыре алгоритма, приводящих к нетипичному мизесовскому преобразованию. В этих алгоритмах зонд сначала начинает движение с места внедрения вправо (к концу пос-ти), до обнаружения первой инверсии. Затем зонд заново устанавливается в позицию своего внедрения и начинает движение влево (к началу пос-ти), до обнаружения первой инверсии.

Два алгоритма (из четырёх) копируют последовательность событий из зондовой области материнской пос-ти (сохранят нумерацию эл зондовой системы координат). Один алгоритм (из двух) копирует зондовую область эл в дочернюю пос-ть вместе с обнаруженными инверсными элами, второй без инверсных эл.

Два других алгоритма (из четырёх) делают то же самое, но без сохранения порядка следования эл присущей зондовой области. Каждый новый эл попадающий в скользящее окно тут же переносится в дочернюю пос-ть. Один алгоритм (из двух) копирует зондовую область эл в дочернюю пос-ть вместе с обнаруженными инверсными элами, второй без инверсных эл.

Зависимость числа найденных спектров от: вида спектра, способа его поиска. Законы вероятности отменить нельзя, поэтому многочисленные события (например, много красных шаров в мешочке) имеют большую вероятность обнаружения, а малочисленные события (например, зелёные шары в том же мешочке) имеют меньшую вероятность обнаружения. Именно разностью в численностях двух конкурирующих поисковых шаблонов в бинарной последовательности, искомых по правилам игры Пенни, объясняется таблица с вероятностями выигрышей для них. Ранее совершенно крамольное утверждение, что «число найденных спектров в бинарной случайной пос-ти зависит от: вида спектра и от способа поиска спектра в пос-ти» - теперь потеряло свою возбуждающую силу, став обыденной истиной, получив своё объяснение. В Пенни подобных способах поиска шаблонов возникают такие условия, которые делают это утверждение истинным: таблица 1, работа [7]. В [5, 6] приведены и другие способы поиска, которые приводят к другим, не стандартным, с позиций доминирующей теории вероятности, результатам.

Все искомые серии (шаблоны) являются логически создаваемой человеком абстракцией. Шаблоны (абстракции), подчиняются логическим правилам их выделения из ряда существующих элементарных событий (эл) пос-ти (правила игры - скользящего окна, правила фрагментирования на отрезки равной длины). Любые способы $[5,6]$ обработки (классификации) пос-ти выпадающих нулей и единиц являются логическими правилами. И из этого множества возможных правил нельзя выбрать одно, более фундаментальное, чем другие, правило. А по разным правилам учёта возникают разные результаты (вероятности), признавать или не признавать которые есть дело воли каждого отдельного человека. Объективная реальность с выпадением монет такова, что она - субъективна, относительна и зависит от системы учёта.

Приведём ещё несколько совершенно крамольных, но не менее эффектных, простых экспериментальных открытий сделанных в рамках «Комбинаторики длинных последовательностей». Алгоритм программы и описание экспериментов даны в [1, 2, 3, 8].

Неожиданная асимметрия 1. Мы все привыкли к мысли, что чётных и нечётных составных событий при подбрасывании монеты должно быть поровну («1», «0», «11», «00», «111», «000», «1111», «0000», ...). Это не так. Нечётных составных событий больше при их последовательном выделении из серии выпадений монеты. Ф.3 [1-3] связывает число составных событий, длины $\square$, с числом эл пос-ти N:

$$
\square=\frac{\square}{2^{\square+1}}
$$

Поскольку по ф.3 самыми многочисленными составными событиями являются события единичной длины ${ }^{l} \square$ («1», «0»), то это и определяет численное преимущество встречаемых нечётных серий при подбрасывании монеты над чётными сериями.

Ещё большей числовой асимметрии подвержены цуги - цепочки, образуемые составными событиями одинаковой длины. Например: «01010», «11001100», «111000». Отметим, что составное событие, окружённое спереди и сзади (слева и справа) составными событиями других длин, тоже является цугой, вернее единичной цугой, пример: «11111», «101111001».

Неожиданная асимметрия 2. Сравнение численностей составных событий и цуг приводит к ещё одному факту, в который просто так поверить нельзя. Так в популярных лекциях по вероятностям, лектор любит задавать вопрос, о том, что чаще встречаются события: «0000000000», «1111111111» или чередования «1010101010», «0101010101». Все дружно отвечают, что одинаково. И это не правильно для подразумевающейся по умолчанию поисковой модели (последовательно просматриваем все результаты выпадений монеты), цуг («гребёнок») указанной длины в четыре раза будет найдено меньше, чем составных событий.

Почему раньше этого не замечали? Потому что искали при помощи других поисковых правил (которые не требуют компьютеров). Когда длинная случайная последовательность из нулей и единиц 
разрезается на отрезки длиной по десять событий, то количества фрагментов с цугами (цуги: «1010101010», «0101010101») будет равно количеству фрагментов с составными событиями («0000000000», «1111111111»). И лектору, из раздела «введение», прежде чем задавать вопрос о том, каких цепочек больше, надо уточнять каким образом он производит поиск этих цепочек.

Очень простые отношения. В любой случайной последовательности из $\square$ результатов подбрасываний монеты будет $\square / 2$ составных событий и $\square / 3$ цуг, $\square / 4$ - число всех последовательных составных событий [1], $\square / 8$ - число всех инверсных составных событий [1].

\section{Выводы}

В статье представлен способ управления и «тонкий» способ управления вероятностью обнаружения (выпадения) серии событий, реализованный на эффекте экранирования, возникающий при использовании правил игры Пенни.

Существуют разные способы поиска шаблона в бинарной пос-ти (разные правила игр), результаты поиска которых раньше расценивались как равновероятные, но на деле приводящие к различным вероятностям обнаружения (выпадения) искомых серий.

В статье показано, что при раздельном поиске (без конкуренции шаблона с другими шаблонами) по пенни подобным правилам, шаблоны с разным содержанием инверсий внутри себя, выпадают с разной частотой (вероятностью). Численность раздельно найденных в пос-ти шаблонов по правилам игры Пенни связана с числом инверсий внутри шаблона.

Шаблоны с одинаковым числом инверсий имеют одинаковое число встреч (равную вероятность выпадения) при раздельном поиске по правилам Пенни внутри пос-ти.

Шаблоны с разным числом инверсий внутри себя, имеют разную частоту встреч (разную вероятность выпадения) в бинарной пос-ти при их раздельном поиске по правилам игры Пенни.

Формально соблюдая требования мизесовских преобразований, которым не противоречит получение информации об одной инверсии в конце области зондирования, можно изменить параметры дочерних пос-тей, которые обретают другой закон распределения для составных событий и цуг, отличный от закона распределения в материнской последовательности. То есть, существует не типичное мизесовское преобразование. Следовательно, инверсии обладают неким, ещё не познанным, скрытым информационным потенциалом.

Открытие зависимости частоты обнаружения искомой серии (шаблона) от способа её поиска и её вида - является самым значимым открытием в современной теории вероятности.

Зависимость вероятности обнаружения от способа поиска и вида серии в последовательности равновероятных бинарных событий является большой философской и статистической проблемой, требующей переосмысления господствующих научных позиций и ряда задач в учебниках.

\section{Лumepamypa}

1. Филатов О. В., Филатов И. О., Макеева Л. Л. и др. Потоковая теория: из сайта в книгу. Москва. «Век информации», 2014. С. 200.

2. Филатов О.В., Филатов И.О. «Закономерность в выпадении монет - закон потоковой последовательности». Германия. Издательский Дом: LAPLAMBERT Academic Publishing, 2015. C. 268.

3. Филатов О.В., Филатов И.О. Статья «О закономерностях структуры бинарной последовательности», «Журнал научных публикаций аспирантов и докторантов». № 5, 2014.

4. Филатов О. В., Филатов И. О. Статья «О закономерностях структуры бинарной последовательности (продолжение)», «Журнал научных публикаций аспирантов и докторантов». № 6, 2014.

5. Филатов О. В. Статья «Описание схем управления вероятностью выпадения независимых составных событий», «Проблемы современной науки и образования». № 2 (44), 2016 г.

6. Филатов О.В. Статья «The use of geometric probability to change the probability of finding a series of random deposition coins. / Применение геометрической вероятности для изменения вероятности нахождения серий случайных выпадений монеты», журнал «Проблемы современной науки и образования / Problems of modern science and education». № 22 (64), 2016 г.

7. Филатов О.В. Статья «Расчёт численностей поисковых шаблонов в парадоксе Пенни», «Проблемы современной науки и образования». № 11 (41), 2015 г.

8. Филатов О. В. Статья «Derivation of formulas for Golomb postulates. A method for creating pseudorandom sequence of frequencies Mises. Basics "Combinatorics of long sequences." / Вывод формул для постулатов Голомба. Способ создания псевдослучайной последовательности из частот Мизеса. Основы «Комбинаторики длинных последовательностей», журнал «Проблемы современной науки и образования / Problems of modern science and education». № 17 (59), 2016 г.

9. Авторский сайт со статьями: http://kodpi.net/ 